\title{
Haptic Assessment in Fabrics with Kansei Evaluation
}

\author{
Nazlina Shaari \\ Design Technology Department, faculty of Applied \& Creative Arts, \\ Universiti Malaysia Sarawak, Malaysia \\ snazlina@faca.unimas.my
}

\begin{abstract}
Today's consumers demand more of what will enrich their life. They desire new products that could fulfill their needs. With the reference to the changing society and consumer -oriented trends, design today takes place in a different framework than ever before. This is where human perceptive faculties consist of five senses: visual, auditory, olfactory, taste, and tactile sense are involved. Visual is the most powerful sense for human perception followed by the auditory sense. This article attempts to find the connection between the visual and the auditory senses. In particular, the aim is to evaluate tactile evaluation, involved with the haptic experience in identifying the preferable materials for clothing. The research will propose methods in Kansei to facilitate the understanding of the different types of haptic activities involved in this testing. These structures clearly recognize and evoke specific elements in fabrics appearances and its semantic values.
\end{abstract}

Keywords-component; fabric, haptic, kansei engineering

\section{INTRODUCTION}

Creativity and Kansei involved the process of thinking and producing. It brings to our awareness that creativity requires passion and commitment. A product is creative when it is novel, original and appropriate. Creativity does stimulate further work and ideas to set better output for product appearance and used. Creativity fostered in design cultures that involved design thinking, and it processes. The role of designers in the industry has expanded from the initial stage of planning the design to the finalization of the function, analysis and evaluation of product feedback. Accordingly, the value of products has changed and users now expect products, which are more approachable, fitting well with their subjective expectation. One feasible way of building sustainable creativity is to understand the Kansei by evaluating human senses and experimenting the processes that are related to products.

Kansei is a Japanese term, which means psychological feeling or image of a product. Kansei Engineering (KE) refers to the translation of consumers' psychological feeling about a product into perceptual design elements, (Nagamachi, 1989). Kansei Engineering (KE) sometimes referred to as "sensory "or "emotional usability." Emotion elicited hedonic values in the product design. This technique involves determining which sensory attributes elicit particular subjective responses from people, and then designing a product using the attributes, which elicit the desired responses, (Nagamachi 1994). This research presents a way of evaluating textiles using Kansei methods. It provides an important contribution towards formation of creativity and Kansei which mapping the behavior-based creativity of the user.

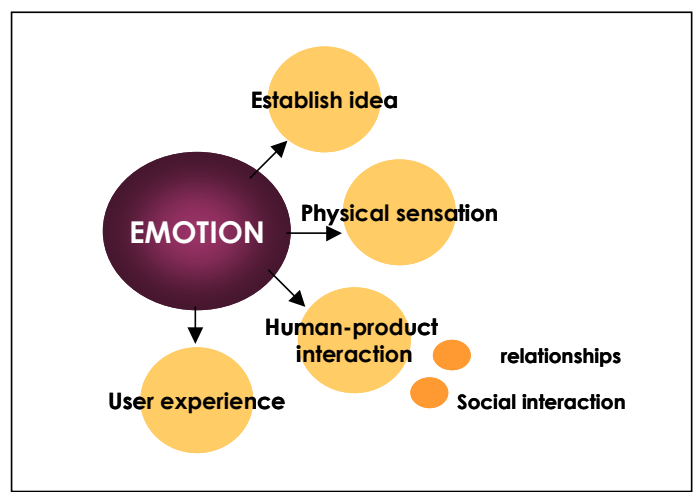

Figure 1: Emotion values in product design

\section{KANSEI EVALUATION}

Kansei Engineering (KES) is a technique for product development (Nagamachi 1995), which takes into account the desirable features of products, as perceived by end users themselves. The technique involves a database of the keywords, which represent consumers' feelings towards products, and then these is used to produce scales, which are then used to evaluate a number of products. A factor analysis technique used to identify those features of product design that correlate with consumers' feelings. The technique also developed to produce design rules for the development of products, and in the evaluation of prototype design

With these considerations, the product such as textiles found closely related to consumers' feeling. To understand the textiles and its relation to the body therefore involves a forever provisional and constantly re-negotiable grasp of activities of seeing and making sense as social practices, largely dedicated by concepts of history, memory, permanent and impermanent, fluctuation in functions and value and generating transitive sense of pleasure and desire.

\section{BEHAVIOR BASE APPROACH}

To study users' perceptions in evaluating fabrics for clothing, KE type 1 are commonly used to generate perceptual 